\title{
Full spin flipping in the presence of full Siberian Snake
}

\author{
M. Bai and T. Roser \\ Brookhaven National Laboratory, Upton, New York 11973, USA
}

(Received 8 June 2007; published 11 September 2008)

\begin{abstract}
This paper proposes a new design of spin flipper for high-energy accelerators to obtain full spin flip with the spin tune staying at half integer. The traditional technique of using a single rf spin rotator with a fixed spin rotation axis and an oscillating spin rotation, either an rf dipole or solenoid, as spin flipper to achieve full spin flip in the presence of a full Siberian snake has been demonstrated [B. B. Blinov et al., Phys. Rev. Lett. 81, 2906 (1998); B. B. Blinov et al., Phys. Rev. Lett. 88, 014801 (2001); V. S. Morozov et al., Phys. Rev. ST Accel. Beams 7, 024002 (2004); R. A. Phelps, AIP Conf. Proc. 338, 361 (1994); Ya. S. Derbenev et al., Part. Accel. 8, 115 (1978)]. However, this technique requires one to change the snake configuration to move the spin tune away from half integer which is not practical for an operational high-energy polarized proton collider such as RHIC where beam and polarization lifetime are sensitive to small changes. Based on the conceptual designs to use rf dipoles to achieve full spin flip with the spin tune at half integer [T. Roser, BNL Report No. BNL-52453, 1994; R. A. Phelps, BNL Report No. BNL-52453, 1994], this paper presents a compact design of a spin flipper for a high-energy accelerator. The theory of the new spin flipper technique and numerical simulations are also presented.
\end{abstract}

DOI: 10.1103/PhysRevSTAB.11.091001

PACS numbers: $29.27 . \mathrm{Hj}$

\section{INTRODUCTION}

In order to cancel the systematic errors in experiments of high-energy polarized proton collisions, one would like to reverse the spin directions of the two colliding beams multiple times during the store when data are taken. In general, a high-energy polarized proton collider employs full Siberian snakes to avoid the polarization loss during the acceleration and store. A full Siberian snake is a device to rotate the spin vector around an axis in the horizontal plane by $180^{\circ}$ [1]. For the Relativistic Heavy Ion Collider (RHIC) at Brookhaven National Laboratory, two pairs of full Siberian snakes were installed in the two rings. In each ring, the two snakes are located $180^{\circ}$ from each other with their spin precession axes perpendicular to each other as shown in Fig. 1. This configuration yields a spin tune $Q_{s}=$ $\frac{1}{2}$ [2] and a vertical stable spin direction around the ring. Here, spin tune is defined as the number of spin precessions in one orbital revolution. Since the betatron tunes in circular accelerators are kept away from half integer to keep the beam stable, both intrinsic and imperfection depolarizing spin resonances at $G \gamma=k P \pm Q_{y}$ and $G \gamma=k$ are avoided [3]. Here, $G$ is the anomalous $g$ factor, $\gamma$ is the Lorentz factor, $k$ is an integer, $P$ is the superperiodicity of the accelerator, and $Q_{y}$ is the vertical betatron tune.

The traditional spin flipping technique uses an rf spin rotator that rotates the spin around an axis in the horizontal plane. Such an rf spin rotator can be implemented as an rf solenoid or an rf dipole with horizontally oriented magnetic field. In the latter case, the effect of the vertical orbit oscillation needs to be considered as well which will be done in Sec. III. For now, only a local rf spin rotator is considered that does not affect the beam orbit. Also, with- out loss of generality, the spin rotation axis is assumed to be radial. To achieve spin flipping, the rf spin rotator frequency is swept adiabatically across the beam spin precession frequency. In this case, the spin precession equation is [4]

$$
\frac{d \vec{S}}{d \theta}=\left[G \gamma \hat{y}+\phi_{\mathrm{osc}}(\theta) \cos \left(Q_{\mathrm{osc}} \theta+\chi\right) \hat{x}\right] \times \vec{S},
$$

where $Q_{\text {osc }}$ is the rf spin rotator tune, which is the oscillating frequency of the rf spin rotator field in units of the particle's revolution frequency. $d \theta=\frac{v}{\rho} d t$, where $v$ is the speed of the particle and $\rho$ is the curvature of the bending dipole. $\chi$ is the initial phase of the oscillating field and is a function of the beam particle's azimuthal position at the beginning of the rf spin rotator excitation. For two particles at two different azimuthal locations, the difference of the initial phases $\Delta \chi$ is

$$
\Delta \chi=Q_{\mathrm{osc}} \Delta \theta,
$$

where $\Delta \theta$ is the azimuthal separation of the two particles. $\phi_{\text {osc }}(\theta)$ is the amount of spin rotation from the rf spin rotator located at $\theta_{\text {osc }}$ :

$$
\phi_{\mathrm{osc}}(\theta)=\sum_{n=-\infty}^{\infty} \tilde{\phi}_{\mathrm{osc}} \delta\left(\theta-\theta_{\mathrm{osc}}-2 \pi n\right),
$$

where $n$ is the orbital revolution number and for the $i$ th turn,

$$
\int_{2 \pi(i-1)}^{2 \pi i} \sum_{n=-\infty}^{\infty} \delta\left(\theta-\theta_{\mathrm{osc}}-2 \pi n\right) d \theta=1 .
$$

Here, $\tilde{\phi}_{\text {osc }}$ is the maximum spin rotation. 


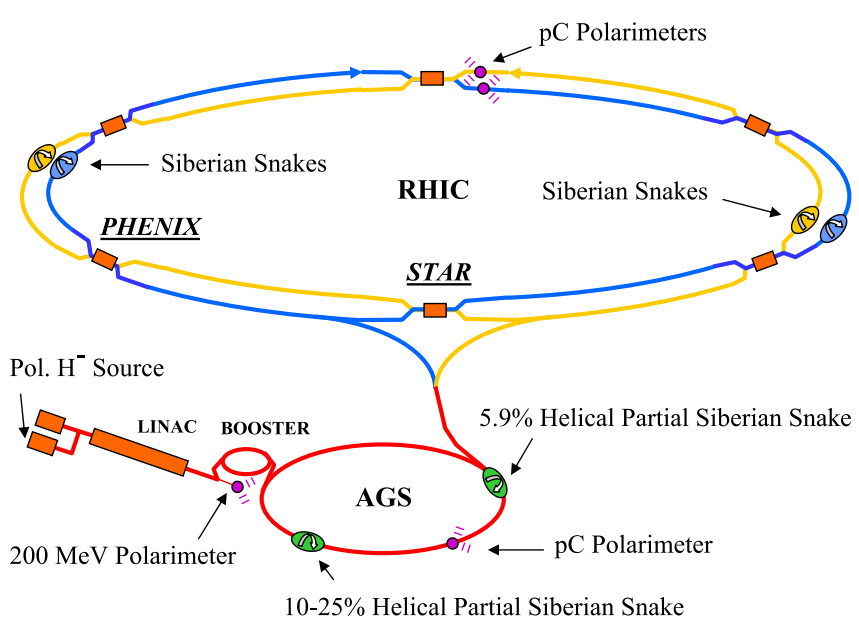

FIG. 1. (Color) This is a schematic layout of the RHIC polarized proton acceleration complex. The polarized $H^{-}$beam from the source gets preaccelerated in the Brookhaven $200 \mathrm{MeV}$ linear accelerator (LINAC) and stripped injected in the Brookhaven Booster. The polarized proton beam is then further accelerated in the Brookhaven AGS and gets injected in the two accelerators of RHIC. The polarized proton acceleration in the $200 \mathrm{MeV}$ LINAC is spin transparent. In the Booster, only 3 imperfection spin resonances are crossed during the acceleration and they are corrected with the vertical harmonic orbit correctors [11]. In the AGS, the polarized beam experiences a total of 7 strong intrinsic spin resonances, 41 imperfection resonances, and 82 horizontal resonances [12] before it gets extracted into RHIC. Two partial helical snakes [13] are employed to preserve the beam polarization [14]. In RHIC, two pairs of full Siberian snakes are employed in the two accelerators to preserve the beam polarization. In order to obtain a vertical stable spin direction in the two accelerators, the two snakes are placed $180^{\circ}$ apart with their spin precession axes perpendicular to each other. This also gives half integer spin tune at all the energies which keeps the beam away from all the intrinsic spin resonances and imperfection spin resonances.

The corresponding spinor equation is

$$
\begin{aligned}
\frac{d \psi(\theta)}{d \theta}= & -\frac{i}{2}\left[G \gamma \sigma_{3}+\phi_{\mathrm{osc}}(\theta)\right. \\
& \left.\times \frac{e^{i\left(Q_{\mathrm{osc}} \theta+\chi\right) \sigma_{3}}+e^{-i\left(Q_{\mathrm{osc}} \theta+\chi\right) \sigma_{3}}}{2} \sigma_{1}\right] \psi(\theta)
\end{aligned}
$$

with

$$
\begin{gathered}
\sigma_{1}=\left(\begin{array}{ll}
0 & 1 \\
1 & 0
\end{array}\right), \quad \sigma_{2}=\left(\begin{array}{cc}
0 & -i \\
i & 0
\end{array}\right), \\
\sigma_{3}=\left(\begin{array}{cc}
1 & 0 \\
0 & -1
\end{array}\right) .
\end{gathered}
$$

Equation (5) shows that the spin kick from the rf spin rotator consists of two terms, $\phi_{\text {osc }}^{+}(\theta)=$ $\frac{\phi_{\text {osc }}(\theta)}{2} e^{-i\left(Q_{\text {osc }} \theta+\chi\right) \sigma_{3}}$ and $\phi_{\text {osc }}^{-}(\theta)=\frac{\phi_{\text {osc }}(\theta)}{2} e^{i\left(Q_{\text {osc }} \theta+\chi\right) \sigma_{3}}$. The two terms drive spin resonances at $Q_{s}=Q_{\text {osc }}$ and $Q_{s}=$ $-Q_{\mathrm{osc}}$. When $Q_{\mathrm{osc}}$ is sufficiently different from $\frac{1}{2}$, in a frame that rotates with $Q_{\text {osc }}$ the spin kick contributions from $\phi_{\mathrm{osc}}^{+}(\theta)$ remain constant, whereas the contributions from the $\phi_{\text {osc }}^{-}(\theta)$ term oscillate at $2 Q_{\text {osc }}$ and adiabatically average to zero over many turns. Hence, this becomes an isolated resonance condition and a full spin flip can be achieved by adiabatically ramping the $\mathrm{rf}$ spin rotator tune $Q_{\text {osc }}$ through $Q_{s}$ [5]. This is shown in Fig. 2 as the red line where the turn-by-turn vertical component of the spin vector was calculated with a spin tune $Q_{s}=0.47$ and a single $\mathrm{rf}$ spin rotator. The maximum spin rotation of the $\mathrm{rf}$ spin rotator was adiabatically ramped from zero to $2.3 \mathrm{mrad}$ in the first 6000 turns at a fixed tune of $Q_{\text {osc }}=$ 0.46 . The $\mathrm{rf}$ dipole tune was then adiabatically swept from 0.46 to 0.48 . This brought the spin vector from the initially vertical up direction to the vertical down direction.

However, this isolated resonance crossing condition does not hold anymore when $Q_{s}=\frac{1}{2}$. When $Q_{\text {osc }}$ adiabatically ramps across $Q_{s}=\frac{1}{2}$, the polarized beam simultaneously crosses the spin resonance at $Q_{s}=Q_{\text {osc }}$ from one side and the spin resonance at $Q_{s}=-Q_{\text {osc }}$ from the opposite side. At $Q_{s}=Q_{\mathrm{osc}}=\frac{1}{2}$, the contribution from the $\phi_{\text {osc }}^{-}(\theta)$ term can cancel the contribution from the $\phi_{\text {osc }}^{+}(\theta)$ term depending on the initial phase $\chi$. Thus, a full spin flip cannot be achieved by adiabatically ramping the rf spin rotator tune $Q_{\text {osc }}$ through the beam spin tune $Q_{s}$. This is evident from the spin tracking shown in Fig. 2 as the green

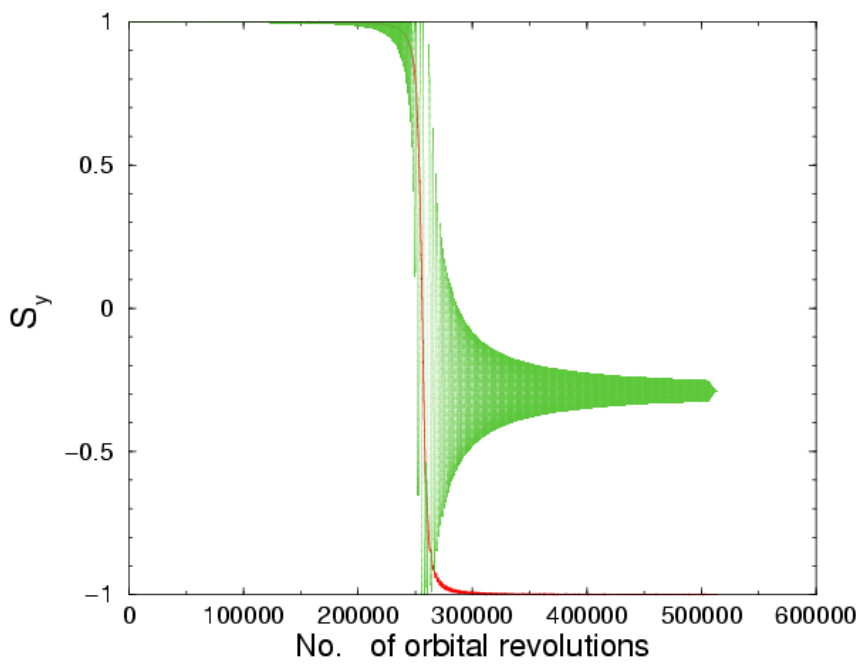

FIG. 2. (Color) This plot shows the spin tracking results of spin flipping using a single rf spin rotator. For the two data sets (the red line and the green line) in this plot, the vertical component of the spin vector is shown as a function of the number of orbital revolutions. In both cases, the initial spin vector points vertically and the maximum spin rotation of the rf spin rotator is adiabatically ramped from zero to $2.3 \mathrm{mrad}$ in the first 6000 turns at a fixed tune of $Q_{s}-0.01$. The rf spin rotator tune was then adiabatically swept from $Q_{s}-0.01$ to $Q_{s}+0.01$ in 500000 turns and then adiabatically ramped down to zero in another 6000 turns. The red line shows full spin flip when the spin precession tune $Q_{s}$ is set to 0.47 and the green line shows depolarization for a spin tune of 0.5 . 
line where all the parameters are the same as in the case shown as the red line, except that the spin tune was set to $Q_{s}=\frac{1}{2}$. One could move the spin tune away from $\frac{1}{2}$ for spin flipping by detuning the snake settings. However, in practice, a high-energy collider is operated with very limited space for spin tune and betatron tune changes and is therefore sensitive to any small tune shifts. It is necessary to develop a spin flipper that functions at the operational spin tune of $\frac{1}{2}$.

\section{SPIN FLIPPER}

The key to achieving full spin flipping with the spin tune at $\frac{1}{2}$ is to provide an rf spin rotator that rotates the spin vector by a constant angle $\tilde{\phi}_{\text {osc }}$ around an axis that is itself rotating in the horizontal plane at a frequency of $Q_{\text {osc }}$. This will eliminate the spin resonance at $Q_{s}=-Q_{\text {osc }}$. Similar to Eq. (1), Eq. (7) is the spin precession equation for an $\mathrm{rf}$ spin rotator with a rotating axis in the horizontal plane:

$$
\begin{aligned}
\frac{d \vec{S}}{d \theta}= & {\left[G \gamma \hat{y}+\phi_{\mathrm{osc}}(\theta)\left[\cos \left(Q_{\mathrm{osc}} \theta+\chi\right) \hat{x}\right.\right.} \\
& \left.+\sin \left(Q_{\mathrm{osc}} \theta+\chi\right) \hat{z}\right] \times \vec{S} .
\end{aligned}
$$

The corresponding spinor equation is then given by

$$
\frac{d \psi(\theta)}{d \theta}=-\frac{i}{2}\left[G \gamma \sigma_{3}+\phi_{\mathrm{osc}}(\theta) e^{-i\left(Q_{\mathrm{osc}} \theta+\chi\right) \sigma_{3}} \sigma_{1}\right] \psi(\theta) .
$$

Transforming Eq. (8) into the frame that rotates at the same frequency as the rf spin rotator frequency, namely

$$
\Psi(\theta)=e^{(i / 2)\left(Q_{\mathrm{osc}} \theta+\chi\right) \sigma_{3}} \psi(\theta),
$$

yields

$$
\frac{d \Psi(\theta)}{d \theta}=-\frac{i}{2}\left(G \gamma-Q_{\mathrm{osc}}\right) \sigma_{3} \Psi(\theta)-\frac{i}{2} \phi_{\mathrm{osc}}(\theta) \sigma_{1} \Psi(\theta) .
$$

The corresponding spinor transfer matrix of the $\mathrm{rf}$ spin rotator with a rotating axis in Eq. (7) is

$$
M_{\text {rot }}=e^{-(i / 2) \tilde{\phi}_{\mathrm{osc}}\left[\cos \left(Q_{\mathrm{osc}} \theta+\chi\right) \sigma_{1}+\sin \left(Q_{\mathrm{osc}} \theta+\chi\right) \sigma_{2}\right]} .
$$

In practice, the spin flipper using the $\mathrm{rf}$ spin rotator with a rotating axis in Eq. (7) can be approximated by placing a constant-field spin rotator with a vertical spin precession axis between two rf spin rotators with fixed rotation axes and oscillating spin rotations. The spin transfer matrix through such a spin flipper is given by

$$
\begin{aligned}
M_{\text {sflip }}= & e^{-(i / 2) \tilde{\phi}_{\text {osc }} \cos \left(Q_{\text {osc }} \theta+\chi_{2}\right) \sigma_{1}} e^{-(i / 2) \phi_{0} \sigma_{3}} \\
& \times e^{-(i / 2) \tilde{\phi}_{\text {osc }} \cos \left(Q_{\text {osc }} \theta+\chi_{1}\right) \sigma_{1}},
\end{aligned}
$$

where $\phi_{0}$ is the amount of spin rotation from the spin rotator and $\chi_{1,2}$ are the initial phases of the two rf spin rotators with fixed axes, respectively. One can prove that for a small $\tilde{\phi}_{\text {osc }}$ and

$$
\chi_{1}-\chi_{2}=180^{\circ}+\phi_{0}
$$

$M_{\text {sflip }}$ in Eq. (12) is equal to

$$
\begin{aligned}
M_{\text {sflip }}= & e^{-(i / 2) \phi_{0} \sigma_{3}} \\
& \times e^{-(i / 2) \tilde{\phi}_{\text {osc }} \sin \phi_{0}\left[\sin \left(Q_{\text {osc }} \theta+\chi_{2}\right) \sigma_{1}-\cos \left(Q_{\text {osc }} \theta+\chi_{2}\right) \sigma_{2}\right]} .
\end{aligned}
$$

For $\chi_{2}=\chi+\frac{3}{2} \pi$, the oscillating spin rotation term in Eq. (14) is then identical to Eq. (11). The constant rotation term $e^{-(i / 2) \phi_{0} \sigma_{3}}$ does not contribute to the spin flipping. Equation (14) also shows that in principle, a full spin flip can be achieved for any $\phi_{0} \neq 0$ and the effective maximum spin rotation of the rf spin rotator becomes

$$
\tilde{\phi}_{\text {osc }} \sin \phi_{0} \text {. }
$$

For $\phi_{0}=90^{\circ}$, the required $\mathrm{rf}$ spin rotation $\tilde{\phi}_{\text {osc }}$ is most effective and Eq. (14) becomes

$$
M_{\text {sflip }}=e^{-(i / 2)(\pi / 2) \sigma_{3}} e^{(i / 2) \tilde{\phi}_{\mathrm{osc}}\left[\sin \left(Q_{\mathrm{osc}} \theta+\chi_{2}\right) \sigma_{1}+\cos \left(Q_{\mathrm{osc}} \theta+\chi_{2}\right) \sigma_{2}\right]} \text {. }
$$

In a real machine, additional spin rotations around the vertical direction have to be added to cancel the extra $\phi_{0}$ rotation in Eq. (14) in order to keep the spin tune unchanged. Figure 3 shows the schematic drawing of spin flipper for RHIC with two rf dipoles and a $90^{\circ}$ spin rotator in between. Two $45^{\circ}$ spin rotators are arranged on either side of the two rf dipoles to keep the spin tune unchanged. With the two snakes in each ring, the spinor one turn map of RHIC in the presence of the spin flipper is given by

$$
\begin{aligned}
\text { OTM }= & \left(-i \sigma_{2}\right) e^{-(i / 2) G \gamma \pi \sigma_{3}}\left(-i \sigma_{1}\right) e^{-(i / 2) G \gamma \pi \sigma_{3}} e^{(i / 2)(\pi / 4) \sigma_{3}} \\
& \times M_{\text {sflip }} e^{(i / 2)(\pi / 4) \sigma_{3}}
\end{aligned}
$$

This arrangement not only provides a solution for spin flipping, but also minimizes the effect on the horizontal closed orbit. Figure 4 shows the result of spin tracking with this simple lattice. It shows a full spin flip when $Q_{\text {osc }}$ is swept from 0.49 to 0.51 . The maximum spin rotation of the two rf spin rotators is $1.14 \mathrm{mrad}$. For high-energy polarized protons, a $90^{\circ}$ spin rotator needs a dipole field of 2.7 Tesla-

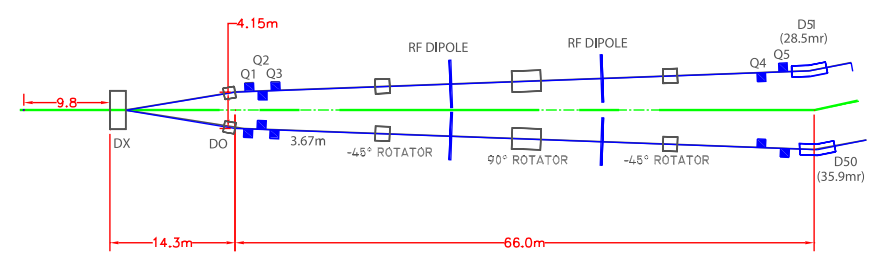

FIG. 3. (Color) The top view schematic layout of the RHIC spin flippers. This shows an available straight section of the RHIC lattice indicating the location of dipoles (DX, D0, D5I, and D5O) and quadrupoles $\left(Q_{i}, i=1,2 \ldots 5\right)$. The separate spin flippers in the two accelerators allow independent spin flipping in each ring. 


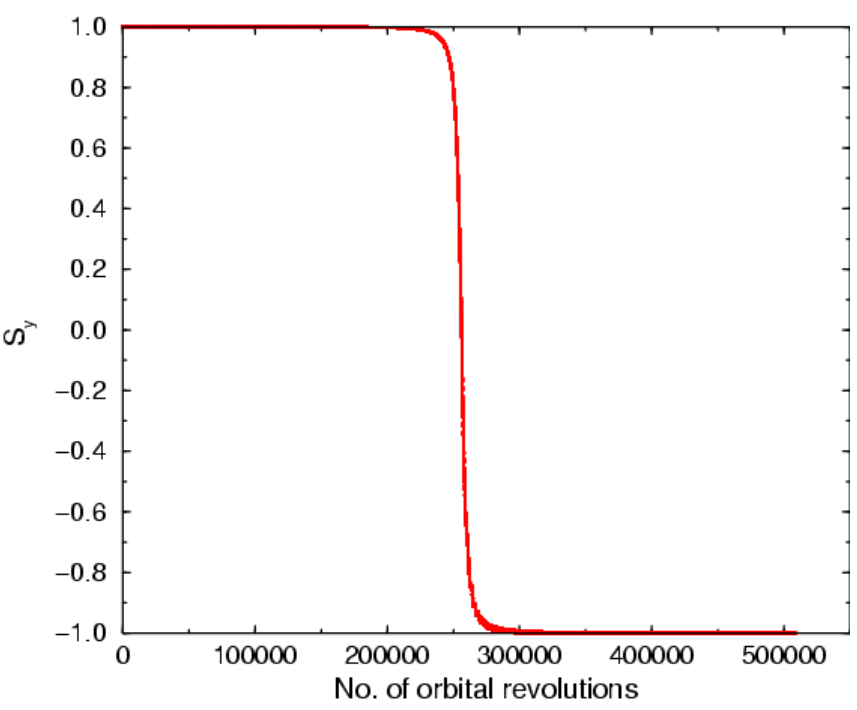

FIG. 4. (Color) This plot shows the vertical component of the spin vector as a function of number of orbital turns for spin tracking done with a simple lattice of two snakes separated by $180^{\circ}$ orbital deflections. The maximum spin rotation of each of the two $\mathrm{rf}$ spin rotators is $1.14 \mathrm{mrad}$ and the initial phase between the two rf dipoles is $90^{\circ}$.

$\mathrm{m}$. This corresponds to a horizontal orbital deflection angle of $8.2 \mathrm{mrad}$ for $100 \mathrm{GeV}$ and $3.2 \mathrm{mrad}$ for $250 \mathrm{GeV}$. These are the typical store energies for RHIC.

Another application of this spin flipper with rotating axis is to measure the spin tune. Instead of sweeping the rf spin rotator tune, one can adiabatically induce a coherent spin precession around the vertical direction by driving the $\mathrm{rf}$ spin rotator at a tune near the spin tune. In this case, the spin vector gets adiabatically moved away from the vertical direction with a constant projection on the vertical direction [3]

$$
S_{y}=\frac{Q_{s}-Q_{\mathrm{osc}}}{\sqrt{\epsilon^{2}+\left(Q_{s}-Q_{\mathrm{osc}}\right)^{2}}},
$$

where $\epsilon=\frac{\tilde{\phi}_{\text {osc }}}{2 \pi}$ is the strength of the spin flipper induced resonance. The projection of the spin vector on the horizontal direction, on the other hand, oscillates with $Q_{\text {osc }}$ and the amplitude of this coherent oscillation is given by

$$
S_{x}=\frac{\epsilon}{\sqrt{\epsilon^{2}+\left(Q_{s}-Q_{\mathrm{osc}}\right)^{2}}} .
$$

The ratio of Eq. (18) and Eq. (19) directly measures the difference between $Q_{s}$ and $Q_{\text {osc }}$ and vanishes at $Q_{s}=Q_{\text {osc }}$. With a fast polarimeter like the RHIC relative carbon polarimeter [6,7], the horizontal and vertical asymmetry (a quantity which is proportional to the spin vector projection on the vertical and horizontal direction) can be recorded on a turn-by-turn basis. The constant vertical asymmetry divided by the Fourier component of the turnby-turn horizontal asymmetry measurement at $Q_{\text {osc }}$ is proportional to the difference between $Q_{s}$ and $Q_{\text {osc }}$. Knowing
$Q_{\text {osc }}$ then gives $Q_{s}$. The advantage of this technique is that this is an adiabatic spin manipulation and can preserve the beam polarization. Hence, this technique is ideal for measuring the spin precession tune at the store energy of a high-energy accelerator.

\section{DRIVEN BETATRON OSCILLATION EFFECT ON SPIN MOTION}

At high energy, an rf spin rotator with fixed axis is realized as an rf dipole magnet. Therefore, in addition to exciting coherent spin precession, an rf dipole with the horizontally oriented magnetic field also induces a coherent betatron oscillation in the vertical plane [8,9]. When the rf dipole is turned on adiabatically at a fixed frequency, the driven betatron oscillation at location $s$ is given by

$$
\begin{aligned}
y_{\mathrm{amp}}(s)= & \sum_{n=-\infty}^{n=\infty} \frac{B_{\mathrm{osc}} L}{2 \pi B \rho} \\
& \times \sqrt{\beta_{y}(s) \beta_{y}\left(s_{\mathrm{osc}}\right)} \frac{Q_{y}}{\left|Q_{y}^{2}-\left(n+Q_{\mathrm{osc}}\right)^{2}\right|},
\end{aligned}
$$

where $s_{\text {osc }}$ is the location of the rf dipole, $B_{\text {osc }} L$ is the amplitude of the integrated field strength of the rf dipole, $Q_{y}$ is the vertical betatron tune, $n$ is an integer, $\beta_{y}(s)$ and $\beta_{y}\left(s_{\text {osc }}\right)$ are the vertical beta functions at the location of the observation and the rf dipole, respectively. The sum of $n$ from $-\infty$ to $\infty$ in Eq. (20) reflects the fact that in principle the localized rf dipole kick excites all the harmonics at $n+$ $Q_{\text {osc }}$. In reality, the driven coherent oscillation is dominated by the harmonic near the betatron tune. For the case when $Q_{\text {osc }}$ is very close to the betatron tune, i.e., $n+$ $Q_{\text {osc }} \simeq Q_{y}$, Eq. (20) becomes [9]

$$
y_{\mathrm{amp}}(s)=\frac{B_{\mathrm{osc}} L}{4 \pi B \rho\left|Q_{y}-\left(n+Q_{\mathrm{osc}}\right)\right|} \sqrt{\beta_{y}(s) \beta_{y}\left(s_{\mathrm{osc}}\right)} .
$$

This driven betatron oscillation introduces additional spin kicks from all the quadrupoles that can either enhance or reduce the strength of the resonance induced by the rf dipole. The spin resonance strength of this driven betatron oscillation is proportional to the nearby intrinsic spin resonance strength (amplitude of the spectral line at $k P \pm Q_{y}$ of the spin perturbations Fourier spectrum [3]) and the amplitude of the driven betatron oscillation given by Eq. (20). For accelerators like RHIC where the spin precession tune is half integer, the rf dipole tune then is required to be swept through half integer for the spin flipping. However, since in general the betatron tune is kept away from half integer for stability reasons, the amplitude of the driven oscillation is insignificant and this effect, as well as the resulting contribution to the rf dipole spin resonance, is negligible. For RHIC, the strength of the strongest intrinsic resonance at the store energy of $G \gamma=$ 191.5 or $G \gamma=477.84$ is about 0.001 for a beam with an 


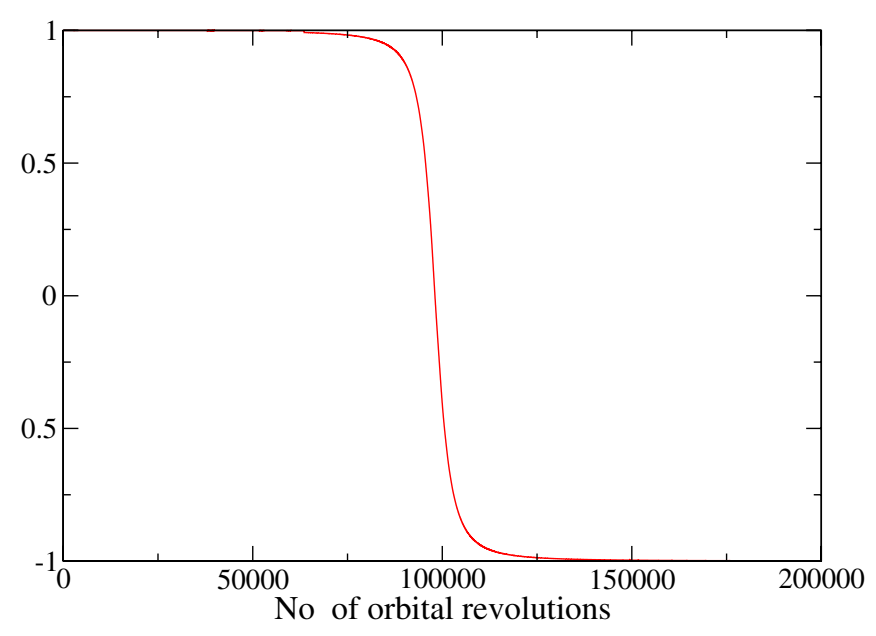

FIG. 5. (Color) This plot shows the vertical component of the spin vector as a function of number of orbital turns for a simulation done using SPINK with the RHIC lattice with all the quadrupoles included. The layout of the spin flipper setup is similar to what is shown in Fig. 3. Instead of a $\phi_{0}=90^{\circ}$ spin rotator in between the two rf dipoles, a $\phi_{0}=30^{\circ}$ spin rotator is used for this tracking and the amplitude of the spin rotation of the two rf dipoles $\tilde{\phi}_{\text {osc }}$ was increased to $2.28 \mathrm{~mm}$-mrad with a phase difference of $\chi_{1}-\chi_{2}=210^{\circ}$.

rms emittance $2.5 \pi \mathrm{mm}$-mrad. The amplitude of the driven oscillation of $\mathrm{rf}$ dipoles with 40 Gauss-m amplitude and $210^{\circ}$ phase difference is about one-tenth of the beam rms size, giving a strength from the driven betatron oscillation of about 0.00003 for a lattice without snakes. This is negligible compared to the effective maximum spin rotation of the two rf dipoles of 0.0012 . With snakes, the effect of driven betatron oscillation on spin motion is further reduced. Figure 5 shows the numerical simulation result with the RHIC lattice with all the quadrupoles included using the spin tracking code SPINK [10] which does the full tracking of both orbital motion and spin motion. Hence, it calculates not only the spin motion directly from the rf dipole, but it also calculates the spin motion from the focusing quadrupole fields that comes from the rf dipole induced coherent betatron oscillation. The full spin flip shown in Fig. 5 confirms that the effect of the driven betatron oscillation on the spin motion is negligible. In this calculation, a $\phi_{0}=30^{\circ}$ spin rotator was chosen in order to use the existing dipole magnets.

\section{CONCLUSION}

This paper presents a new spin flipper design for operational colliders like RHIC where the spin tune is $\frac{1}{2}$. It demonstrates through theoretical derivation as well as numerical simulations that a full spin flip can be achieved without moving the spin tune away from $\frac{1}{2}$. This design can also be used to measure the spin tune in high-energy polarized proton accelerators.

\section{ACKNOWLEDGMENTS}

The authors would like to thank Alfredo Luccio for his strong support on the spin tracking code SPINK and Todd Satogata for his proofreading. We would also like to thank Jim Alduino and Laura Taddonio from the BNL Collider Accelerator department mechanical engineering group for helping to make the schematic drawing of the RHIC spin flipper as shown in Fig. 3. The work was performed under the auspices of the U.S. Department of Energy.

[1] Ya. S. Derbenev and A. M. Kondratenko, Sov. Phys. Dokl. 20, 562 (1976).

[2] RHIC Spin Design Manual (1998).

[3] S. Y. Lee, Spin Dynamics and Snake in Synchrotrons (World Scientific, Singapore, 1997).

[4] M. Bai et al., Phys. Rev. ST Accel. Beams 8, 099001 (2005).

[5] M. Froissart and R. Stora, Nucl. Instrum. Methods 7, 297 (1960).

[6] O. Jinnouchi et al., Proceedings of the 16th International Spin Physics Symposium SPIN2004 (World Scientific, Singapore, 2004), p. 515.

[7] J. Tojo et al., Phys. Rev. Lett. 89, 052302 (2002).

[8] S. Y. Lee, Phys. Rev. ST Accel. Beams 9, 074001 (2006).

[9] M. Bai et al., Phys. Rev. E 56, 6002 (1997).

[10] A. U. Luccio, Spin Tracking in RHIC (Code SPINK) Proceedings of Trends on Collider Spin Physics, ICTP, Trieste, Italy, 1995.

[11] H. Huang, Ph.D. thesis, Indiana University, 1994.

[12] F. Lin et al., Phys. Rev. ST Accel. Beams 10, 044001 (2007).

[13] T. Roser, in Properties of Partially Excited Siberian Snake, in High Energy Spin Physics: 8th International Symposium, Minneapolis, 1988, edited by K. J. Heller, AIP Conf. Proc. No. 187 (AIP, New York, 1989), p. 1442.

[14] H. Huang et al., Phys. Rev. Lett. 99, 154801 (2007). 